\title{
Suicide in the Global Chinese Aging Population: A Review of Risk and Protective Factors, Consequences, and Interventions
}

\author{
XinQi Dong ${ }^{*}$, E-Shien Chang ${ }^{1}$, Ping Zeng ${ }^{2}$, Melissa A. Simon ${ }^{3}$ \\ ${ }^{1}$ Rush Institute for Healthy Aging, Rush University Medical Center, Chicago, Illinois \\ ${ }^{2}$ Peking Union Medical College Hospital, Beijing, China \\ ${ }^{3}$ Feinberg School of Medicine, Northwestern University, Chicago, Illinois
}

[Received January 3, 2014; Revised February 4, 2014; Accepted February 22, 2014]

\begin{abstract}
As one of the leading causes of death around the world, suicide is a global public health threat. In the Chinese population, suicides constitute one-fifth of all recorded suicides in the world. Despite the factual data on suicide rates, the understanding of various causal factors behind suicide, including risk and protective factors and adverse health care, remained incomplete among the global Chinese aging population. To fill in the knowledge void, this paper reviews the epidemiology of suicide among Chinese older adults globally as well as explores the existing intervention strategies. Using the PRISMA statement, we performed a systematic review of exiting research on the topic, including studies describing suicide among Chinese older adults in communities outside of Asia. A literature search was conducted online by using both medical and social science data-bases. Our findings highlighted that elderly suicide in Chinese populations is significantly affected by the social, cultural, and familial contexts within which the individual lived prior to committing suicide. Reviewing such research indicated that while reducing risk factors may contribute to lowering suicides amongst Chinese older adults, measures to improve protective factors are also critical. Support through ongoing family and community care relationships is necessary to improve resilience in older adults and positive aging. Future longitudinal studies on the risk factors and protective factors, and adverse health consequences are called for to devise culturally and linguistically appropriate prevention and intervention programs in global Chinese aging populations.
\end{abstract}

Key words: Psychological distress, older adults, Chinese population

Suicides in the Chinese population constitute one-fifth of all recorded suicides in the world. Evidence suggests that the prevalence of suicide among Chinese older adults approaches, or even surpasses the prevalence estimates in Western countries. A suicide mortality rate of 23 per 100,000 , or 287,000 deaths, is reported annually in China [1]. In addition, suicide is the leading cause of deaths from injury among all aging groups in China, in which the elderly population is particularly susceptible [2]. The Chinese elderly population has a higher prevalence of suicide than the general population. The over- 65 age group has the highest rate of completed suicide, reaching 44.3-
200 per 100,000, which is four to five times higher than the general population [3]. Suicide rates are reported to dramatically increase with age; with the rate peaking for those over 75 years old.

Where China has one of the highest suicide rates in the world, Chinese Americans over the age of 65 have the highest rate of completed suicide in the U.S. over any other racial groups nationwide. Specifically, the suicide rate among Chinese American older women is higher when compared with the general population. A prior study noted a 3 -fold higher suicide rate among U.S. Chinese women aged 65-74 years; 7-fold higher suicide rate

*Correspondence should be addressed to: XinQi Dong, MD, MPH, Medicine, Nursing, and Behavioral Sciences, Rush University Medical Center, Chicago, IL 60612, USA. Email: xinqi_dong@rush.edu 
among the U.S. Chinese women aged 75-84 years; and 10fold higher suicide rate among the U.S. Chinese women over age 85 compared to white women of the same age groups [4].

Despite the factual data on suicide rates, the understanding of various causal factors behind suicide, including risk and protective factors and adverse health care, remained incomplete. In the U.S., Chinese older immigrants have been identified as an underserved group with low acculturation level and high cultural and social isolation. Prior studies suggest that there are significant health disparities among Chinese persons, including chronic diseases, cancer screening and treatment, and psychological distress after having relocated and losing contact with their native support network [5]. In addition, Chinese immigrants experience more pronounced migratory and psychosocial distress, which is further exacerbated by the vast cultural and linguistic barriers, as well as physical frailty and stressful life events [6]. The psychological distress experienced by this population imposes significant health related consequences, and further impedes the quality-of-life of Chinese older adults.

Therefore, this paper aims to illuminate factors specific to the Chinese immigrant aging population through reviewing the epidemiology of suicide amongst this group. Ultimately, this review study aims to bring new insights on cultural and contextual factors unique to Chinese aging populations in the U.S. and worldwide.

\section{Literature Search}

The purpose of this article is to provide a critical review of the research on the epidemiology of suicide among Chinese older adults, including risk and protective factors, health consequences associated with suicidal ideation, attempts and behaviors, as well as to suggest directions for future research and ways to improve care for the Chinese aging population.

The study design was developed according to the Preferred Reporting Items for Systematic Reviews and Meta-Analyses (PRISMA) guidelines. Studies eligible for inclusion in this review were studies assessing suicide risk and protective factors, and adverse health outcomes associated with suicidal behaviors among Chinese older adults in diverse communities, including Chinese elderly in China, as well as overseas Chinese communities. As such, original research articles related to Chinese aging populations around the world were included. The search was limited to studies available in full-text, and written either in English or in Chinese. Manuscripts published over 2 decades ago (pre-1990's) were excluded.

We conducted literature searches online using both medical and social science data bases, including MEDLINE, PubMed, PsychInfo, China journals full-text database (www.cnki.net), Wan Fang data and Google). Search terms used included "suicidal ideation", "suicide attempts", "suicidal behavior", "Chinese", "suicide and elderly", "suicide and older people," in English and "zì sha" ("suicide") in Chinese.

\section{Risk Factors Associated with Suicide}

We reviewed evidence on risk factors for suicidal ideation and suicidal behaviors. Given the inherent diversity among global Chinese communities, we presented the findings according to different geographic regions (Table 1, 2.\& 3).

\section{Demographic Risk Factors}

Research on elderly suicide in China reveals several specific findings. For instance, older age and female gender were associated with increased suicidal behavior. Interestingly, while most studies reported that the risk of suicide increases with age [7], one study in metropolitan China revealed that younger age was associated with higher odds of suicidal ideation [8]. Research also shows that the urban/rural divide poses as a risk factor for suicide; those living in Northern China and in rural areas were found to be at a higher risk of suicide [9]. In addition, social status was found to predispose individuals to a greater risk of suicide and lower education was suggested to increase the risk of suicide. Furthermore, financial constraint is evidenced as a main influencing factor for suicidal ideation and behavior [10].

\section{Psychological and Social Risk Factors}

A significant correlation exists between depressive symptoms, suicide attempts, and suicide mortality among Chinese older adults. Depressive symptoms have been associated with previous suicide attempts. Negative events in family life and social well-being, including a number of stressful events and life changes were also found to be relevant to suicide [11]. In addition, researchers have begun to examine specific forms of psychological distress that are associated with suicidal behaviors. As such, the loss of a partner and feelings of hopeless may both increase distress to a point that a senior person feels that life has become unbearable and in turn, seek suicide.

Regarding social risk factors, the experience of severe interpersonal conflict before death is associated with previous suicidal behavior. Having relatives, friends or associates with suicidal behavior was also found to increases the risks for suicide $[12 ; 13]$. One qualitative study with older veterans in Taiwan reported that family conflict also triggers suicidal behavior [14]. 
Table 1. Risk and Protective Factors Associated with Suicidal Ideation, Suicide Attempts, and Death by Suicide in Chinese Aging Population in Mainland China with Prospective Study or Case Control Study Design.

\begin{tabular}{|c|c|c|c|c|c|c|}
\hline $\begin{array}{l}\text { Author, } \\
\text { Year }\end{array}$ & $\begin{array}{l}\text { Study } \\
\text { Design }\end{array}$ & $\begin{array}{l}\text { Population and } \\
\text { Setting }\end{array}$ & \begin{tabular}{|l|} 
Key \\
Independent \\
Variables
\end{tabular} & Confounding Factors & $\begin{array}{l}\text { Out- } \\
\text { comes }\end{array}$ & Key Findings \\
\hline \multicolumn{7}{|c|}{ Hong Kong } \\
\hline $\begin{array}{l}\text { Sun, } 2011 \\
{[37]}\end{array}$ & PS & $\begin{array}{l}56,088 \text { comm } \\
\text { older adults } 65+\end{array}$ & Dep & $\begin{array}{l}\text { Age, edu, monthly } \\
\text { expenditure, smoking, } \\
\text { alcohol drinking, physical, } \\
\text { activity, body mass index, } \\
\text { health status, self-rated } \\
\text { health }\end{array}$ & SD & $\begin{array}{l}\text { Depressive symptoms were associated with COS } \\
\text { in men } \mathrm{OR}=2.81(1.13-7.01) \text { and women } \\
\mathrm{OR}=2.40(1.18-4.82)\end{array}$ \\
\hline \begin{tabular}{|l} 
Sun, 2011 \\
{$[38]$}
\end{tabular} & PS & $\begin{array}{l}55,946 \text { comm } \\
\text { older adults } 65+\end{array}$ & Dep & $\begin{array}{l}\text { Age, edu, monthly } \\
\text { expenditure, smoking, } \\
\text { alcohol drinking, physical } \\
\text { activity, body mass index, } \\
\text { health status, self-rated } \\
\text { health }\end{array}$ & SD & $\begin{array}{l}\text { Depressive symptoms were associated with } \\
\text { suicide } \\
\text { Men :HR=2.03(0.96-4.29) } \\
\text { Women: HR = 2.36(1.31-4.24). }\end{array}$ \\
\hline $\begin{array}{l}\text { Cheung, } \\
2008[20]\end{array}$ & PS & $\begin{array}{l}\text { Comm data of } \\
\text { older adults } 65+\end{array}$ & SARS & $\begin{array}{l}\text { SDE psychiatric profile, } \\
\text { medical profile and } \\
\text { personal issues }\end{array}$ & SD & 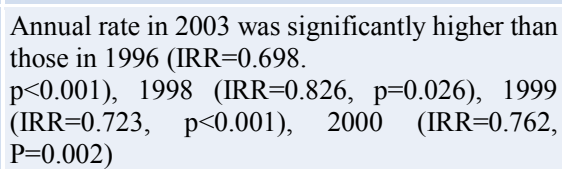 \\
\hline $\begin{array}{l}\text { Lee, } 2007 \\
\text { [39] }\end{array}$ & CS & $\begin{array}{l}1,226 \text { comm } \\
\text { older adults }\end{array}$ & Age & SDE & SI & $\begin{array}{l}\text { Older group }(51-75 \text { years }) \text { were more likely to } \\
\text { consider suicide }(\mathrm{OR}=2.3) \text { than the youngest age } \\
\text { group }(<25 \text { years). }\end{array}$ \\
\hline $\begin{array}{l}\text { Yip,2003 } \\
\text { [40] }\end{array}$ & CS & $\begin{array}{l}917 \text { older adults } \\
60+\end{array}$ & Health Status & SDE & SD & 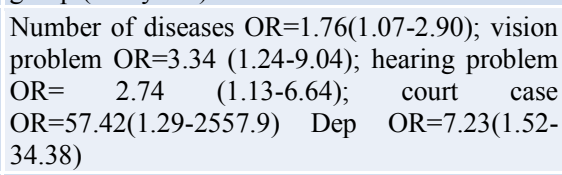 \\
\hline $\begin{array}{l}\text { Chi,1998 } \\
{[25]}\end{array}$ & $\mathrm{CS}$ & $\begin{array}{l}279 \text { comm } \\
\text { suicide cases of } \\
\text { older adults } 60+\end{array}$ & $\begin{array}{l}\text { Gender, severe or } \\
\text { painful illness }\end{array}$ & SDE & SD & $\begin{array}{l}\text { The gender ratio: } 1.4: 1 \text {, severe or painful illness } \\
(80 \%) \text {, from terminal illness }(25 \%) \text {, had a history } \\
\text { of receiving psychiatric treatment } \\
(25 \%) \text {.indicated suicidal idea before their fatal } \\
\text { acts }(68 \%)\end{array}$ \\
\hline $\begin{array}{l}\text { Tsoh, } 2005 \\
\text { [41] }\end{array}$ & $\mathrm{CC}$ & $\begin{array}{l}224 \text { older adults } \\
\text { aged } 65+\end{array}$ & $\begin{array}{l}\text { SDE, } \\
\text { circumstances of } \\
\text { the suicide act, } \\
\text { SA }\end{array}$ & $\begin{array}{l}\text { SDE, Circumstances of } \\
\text { the suicide act and suicide } \\
\text { intent, Psychiatric } \\
\text { morbidities }\end{array}$ & SA & $\begin{array}{l}\text { Current major dep } \mathrm{OR}=59.2(6.4-546.6) \text {, past } \mathrm{SA} \\
\mathrm{OR}=39.1(1.2-1,244.7) \text {, arthritis } \mathrm{OR}=22.6(3.2- \\
\text { 157.3), living with children } \mathrm{OR}=0.2(0.03-0.9) \text {, } \\
\text { conscientiousness } \mathrm{OR}=0.7(0.5-0.9), \\
\mathrm{OR}=0.3(0.1-0.7)\end{array}$ \\
\hline $\begin{array}{l}\text { Chiu,2004 } \\
\text { [42] }\end{array}$ & $\mathrm{CC}$ & $\begin{array}{l}70 \text { suicide cases } \\
\text { and } 100 \text { controls }\end{array}$ & $\begin{array}{l}\text { Mental illness, } \\
\text { history of SA, } \\
\text { health care } \\
\text { utilization }\end{array}$ & SDE & SA & $\begin{array}{l}\text { SA history }: \mathrm{OR}=39.6 \mathrm{p}<0.0001, \text { depressive } \\
\text { disorders } \mathrm{OR}=59.24(20.68-169.65) ; \text { major } \\
\text { depression } \mathrm{OR}=36.25(10.48-125.41)\end{array}$ \\
\hline \multicolumn{7}{|l|}{ Taiwan } \\
\hline $\begin{array}{l}\text { Chan,2011 } \\
{[43]}\end{array}$ & $\mathrm{CS}$ & $\begin{array}{l}3,853 \text { comm } \\
\text { older adults over } \\
65+\end{array}$ & $\begin{array}{l}\text { Gender, income, } \\
\text { smoke, dep, heart } \\
\text { disease }\end{array}$ & $\begin{array}{l}\text { SDE, Physical Disorders, } \\
\text { Pain Symptoms }\end{array}$ & SI & $\begin{array}{l}\text { SI Predictor: Female } \mathrm{OR}=2.27(1.29-4.00) \text {, No } \\
\text { income } \mathrm{OR}=1.68(1.11-2.53) \text {, Former smoker } \\
\mathrm{OR}=2.05(1.09-3.85), \quad \text { Depressive symptoms } \\
\mathrm{OR}=34.30(21.89-\quad 53.76), \text { Heart disease } \\
\mathrm{OR}=1.63(1.06-2.49)\end{array}$ \\
\hline $\begin{array}{l}\text { Yan, } 2009 \\
{[14]}\end{array}$ & $\mathrm{CS}$ & $\begin{array}{l}19 \text { older adults } \\
\text { over } 65+\text { with } \\
\text { SA in veterans' } \\
\text { homes }\end{array}$ & $\begin{array}{l}\text { Reasons that } \\
\text { cause suicide }\end{array}$ & SDE & SI, SA & $\begin{array}{l}\text { Qualitative data indicated five major themes } \\
\text { related to suicide triggers: illness and pain, death } \\
\text { of close relatives or friends, family conflicts, } \\
\text { disputes with friends or workers, and difficulty } \\
\text { adapting to institutional life }\end{array}$ \\
\hline $\begin{array}{l}\text { Yen,2005 } \\
{[44]}\end{array}$ & CS & $\begin{array}{l}1,000 \text { older } \\
\text { adults aged } 65- \\
74\end{array}$ & Suicidal thoughts & $\begin{array}{l}\text { Sex, edu, religious belief, } \\
\text { employment status, marital } \\
\text { status, living } \\
\text { arrangement, and family } \\
\text { monthly income }\end{array}$ & SI & $\begin{array}{l}\text { Dep OR=15.47 }(9.76-24.53) \text {, lower level of edu } \\
\mathrm{OR}=2.04(3.31-1.25), \text { community participation } \\
\text { in the past } 6 \text { months } \mathrm{OR}=1.72(1.07-2.96)\end{array}$ \\
\hline
\end{tabular}

Note: CS: Cross sectional study; PS: Prospective study; CC: Case control study; OR: odds ratio; RR: Relative risk; Hazard ratio; HR: IRR: Incident rate ratio. SA: Suicide attempt; SI: Suicide ideation; SD: Death by Suicide; Com: Community-dwelling; SDE: Sociodemographic variables; Edu: Education, Dep: Depression; QOL: Quality of Life; Manuscripts are ordered according to the year of publication 
Table 2. Risk and Protective Factors Associated with Suicidal Ideation, Suicide Attempts, and Death by Suicide in Chinese Aging Population in Mainland China with Cross Sectional Study Design.

\begin{tabular}{|c|c|c|c|c|c|c|}
\hline $\begin{array}{l}\text { Author, } \\
\text { Year }\end{array}$ & $\begin{array}{l}\text { Study } \\
\text { Design }\end{array}$ & $\begin{array}{l}\text { Population and } \\
\text { Setting }\end{array}$ & $\begin{array}{l}\text { Key Independent } \\
\text { Variables }\end{array}$ & $\begin{array}{l}\text { Confounding } \\
\text { Factors }\end{array}$ & $\begin{array}{l}\text { Out- } \\
\text { comes }\end{array}$ & Key Findings \\
\hline $\begin{array}{l}\text { Rebholz, } \\
\text { 2011(10) }\end{array}$ & PS & $\begin{array}{l}169,871 \text { comm } \\
\text { adults } 40+\text { in } \\
\text { China }\end{array}$ & $\begin{array}{ll}\text { Age, } & \text { gender, } \\
\text { geographic } & \text { region, } \\
\text { urbanization } & \end{array}$ & SDE & SD & $\begin{array}{l}\text { Male }(R R=1.56,1.03-2.38) \text {, aged above } 70(R R=2.27 \text {, } \\
1.29-3.98) \text {, north China }(R R=1.68,1.20-2.36) \text {, rural } \\
(R R=2.82,1.76-4.51) \text { were associated with increased } \\
\text { mortality }\end{array}$ \\
\hline $\begin{array}{l}\mathrm{Xu}, 2000 \\
(33)\end{array}$ & PS & $\begin{array}{l}\text { Comm older } \\
\text { adults } 60+\text { and } \\
\text { older in urban } \\
\text { and rural China }\end{array}$ & Health, family conflict & SDE & SD & $\begin{array}{l}\text { Urban: chronic illness and life embarrassment }(45.3 \%) \text {, } \\
\text { family conflicts }(37.7 \%) \text { and psychiatric disease } \\
(16.9 \%) \text { Rural: family conflicts }(76.3 \%) \text { chronic } \\
\text { illness and life embarrassment }(20 \%) \text {, difficulty of life } \\
(3.5 \%) \text {. }\end{array}$ \\
\hline $\begin{array}{l}\mathrm{Lu}, 2004 \\
(34)\end{array}$ & $\mathrm{CC}$ & $\begin{array}{l}134 \text { rural comm } \\
\text { suicide } \text { older } \\
\text { adults } 60+\text { in } \\
\text { China }\end{array}$ & $\begin{array}{l}\text { Edu, marital status, } \\
\text { economic condition }\end{array}$ & SDE & SD & $\begin{array}{l}\text { Educational level was lower }\left(\mathrm{X}^{2}=16.1, \mathrm{P}=0.001\right) \text {, } \\
\text { partner-lost rate was higher }\left(\mathrm{X}^{2}=5.9, \mathrm{P}=0.015\right) \text {, medical } \\
\text { cost was higher }\left(\mathrm{X}^{2}=7.2, \mathrm{P}=0.03\right) \text {, family income was } \\
\text { lower }\left(\mathrm{X}^{2}=10.0, \mathrm{P}=0.02\right) \text { in accident death elderly.. }\end{array}$ \\
\hline $\begin{array}{l}\text { Tao,2011 } \\
(35)\end{array}$ & $\mathrm{CC}$ & $\begin{array}{l}670 \text { rural comm. } \\
\text { adults } 60+\text { in } \\
\text { Hunan, China }\end{array}$ & $\begin{array}{l}\text { Family status, spousal } \\
\text { relationship, children } \\
\text { relationship, physical } \\
\text { health }\end{array}$ & SDE, social Support & SI & $\begin{array}{l}\text { Source of finance: } \mathrm{OR}=0.59(0.36-0.96) \text {, } \\
\text { children relationship: } \mathrm{OR}=0.31 \quad(0.13-0.72) \text {; physical } \\
\text { health: } \mathrm{OR}=0.17(0.04-0.80)\end{array}$ \\
\hline $\begin{array}{l}\text { Zhang, } \\
2010(36)\end{array}$ & $\mathrm{CC}$ & $\begin{array}{l}454 \text { comm. male } \\
\text { suicides in China }\end{array}$ & $\begin{array}{l}\text { Alcohol use disorder, } \\
\text { Acute alcohol use }\end{array}$ & $\begin{array}{l}\text { Age, marital status, } \\
\text { employment status, } \\
\text { major depression, } \\
\text { prior } \\
\text { functioning, and } \\
\text { acute life events }\end{array}$ & SA & $\begin{array}{l}\text { Alcohol use disorder is more likely to predict previous } \\
\text { SA (OR=1.94, } 1.07-3.53), \text { Age }>60 y \text {, Alcohol use } \\
\text { disorder, Acute alcohol use } \mathrm{OR}=1 \text {. Elderly alcohol: } \\
\text { non-alcohol }=16(23.5 \%): 100(31.9 \%)\end{array}$ \\
\hline $\begin{array}{l}\text { Jun, } \\
2006(37) \\
\end{array}$ & $\mathrm{CC}$ & $\begin{array}{l}915 \text { comm. older } \\
\text { adults } 60+\end{array}$ & Female gender & SDE & SI & $\begin{array}{l}\text { Significant difference between male and female older } \\
\text { adults }\left(\chi^{2}=9.09, \mathrm{P}<0.01\right)\end{array}$ \\
\hline $\begin{array}{l}\text { Phillips, } \\
2002 \text { (13) }\end{array}$ & $\mathrm{CC}$ & $\begin{array}{l}519 \text { comm } \\
\text { suicide adult and } \\
536 \text { controls in } \\
\text { China }\end{array}$ & $\begin{array}{l}\text { Dep, previous SA, } \\
\text { acute stress, QOL, } \\
\text { chronic stress, severe } \\
\text { life event, other's } \\
\text { suicidal behavior }\end{array}$ & $\begin{array}{l}\text { Sex, age, location of } \\
\text { residence, and } \\
\text { geographic site }\end{array}$ & SA, SD & $\begin{array}{l}\text { Dep: score }>37: \mathrm{OR}=41.5(17.2-100.2) \text {, previous SA: } \\
\mathrm{OR}=12.9(4.0-41.0) \text {, acute stress: high score } \\
\mathrm{OR}=9.3(4.2-20.6) \text {, QOL: low score } \mathrm{OR}=7.9(3.7-16.8) \text {, } \\
\text { chronic stress: high score: } \mathrm{OR}=7.6(3.7-15.8) \text {, } \\
\text { Experienced severe life event: } \mathrm{OR}=7.4(3.3-16.3) \text {, } \\
\text { Blood relative had suicidal behavior: } \mathrm{OR}=3.4(1.6-7.2) \text {, } \\
\text { Friends or associates had suicidal behavior: } \\
\mathrm{OR}=3.3(1.9-5.8) \text {. }\end{array}$ \\
\hline $\begin{array}{l}\text { Qin,2002 } \\
\text { (12) }\end{array}$ & $\mathrm{CC}$ & $\begin{array}{l}80 \text { women with } \\
\text { dep aged } 60-82 \text {, } \\
\text { discharged from } \\
\text { a mental disease } \\
\text { hospital }\end{array}$ & $\begin{array}{l}\text { Edu, physical disease, } \\
\text { life event, dep }\end{array}$ & $\begin{array}{l}\text { Age, positive family } \\
\text { history, character, } \\
\text { marital status, } \\
\text { mental disorder }\end{array}$ & SA & $\begin{array}{l}\text { Higher education level }(\mathrm{x} 2=8.83, \mathrm{P}<0.05) \text {, physical } \\
\text { disease }(\mathrm{P}<0.05) \text {, main depression }(\mathrm{P}<0.005) \text {, higher } \\
\text { HAMD score }(\mathrm{P}<0.01)\end{array}$ \\
\hline
\end{tabular}

Note: CS: Cross sectional study; PS: Prospective study; CC: Case control study; OR: odds ratio; RR: Relative risk; Hazard ratio; HR: IRR: Incident rate ratio. SA: Suicide attempt; SI: Suicide ideation; SD: Death by Suicide; Com: Community-dwelling; SDE: Sociodemographic variables; Edu: Education, Dep: Depression; QOL: Quality of Life; Manuscripts are ordered according to the year of publication.

\section{Psychiatric Risk Factors}

The presence of a psychiatric disorder is among the most consistently reported risk factors for suicidal behavior. Researchers have begun to examine more specific constructs that may explain exactly why psychiatric disorders are associated with suicidal behavior. Several such risk factors include mood, anxiety, bi-polar, and other mental disorders $[15 ; 16]$.

\section{Biological Risk Factors}

Genetic association studies have suggested that genes including serotonin transporter, tryptophan hydroxylase
2, and brain-derived neurotrophic factor are related to suicide behaviors. However, most research in Chinese elderly or adult populations did not report the associations and some researchers speculated that the relevance of genetic factors could be ethnicity dependent [17]. When Hwang et al. [18] genotyped APOE in 111 older inpatients diagnosed with major depression and 144 normal controls in Taiwan, he found that depressed subjects who were APOE4 carriers showed a higher suicide attempt history than depressed subjects lacking the APOE epsilon 4 allele $(p=0.012)$. On the other hand, Jia et al. [19] revealed catechol-O-methyltransferase (COMT) Val/Val 158/108 as a susceptible genetic factor for attempted suicides. 
Table 3. Risk and Protective Factors Associated with Suicidal Ideation, Suicide Attempts, and Death by Suicide in Chinese Aging Population in Hong Kong and Taiwan.

\begin{tabular}{|c|c|c|c|c|c|c|}
\hline $\begin{array}{l}\text { Author, } \\
\text { Year }\end{array}$ & \begin{tabular}{|l|} 
Study \\
Design
\end{tabular} & $\begin{array}{l}\text { Population } \\
\text { and Setting }\end{array}$ & \begin{tabular}{|l|} 
Key \\
Independent \\
Variables
\end{tabular} & Confounding Factors & $\begin{array}{l}\text { Out- } \\
\text { comes }\end{array}$ & Key Findings \\
\hline \multicolumn{7}{|l|}{ Hong Kong } \\
\hline \begin{tabular}{|l|} 
Sun, 2011 \\
(41)
\end{tabular} & PS & $\begin{array}{l}56,088 \text { comm } \\
\text { older adults } \\
65+\end{array}$ & Dep & $\begin{array}{l}\text { Age, edu, monthly } \\
\text { expenditure, smoking, alcohol } \\
\text { drinking, physical, activity, } \\
\text { body mass index, health } \\
\text { status, self-rated health }\end{array}$ & SD & $\begin{array}{l}\text { Depressive symptoms were associated with } \mathrm{COS} \text { in } \\
\text { men } \mathrm{OR}=2.81(1.13-7.01) \text { and women } \mathrm{OR}=2.40(1.18 \text { - } \\
4.82)\end{array}$ \\
\hline $\begin{array}{l}\text { Sun, } 2011 \\
(42)\end{array}$ & PS & $\begin{array}{l}55,946 \text { comm } \\
\text { older adults } \\
65+\end{array}$ & Dep & $\begin{array}{l}\text { Age, edu, monthly } \\
\text { expenditure, smoking, alcohol } \\
\text { drinking, physical activity, } \\
\text { body mass index, health } \\
\text { status, self-rated health }\end{array}$ & SD & $\begin{array}{l}\text { Depressive symptoms were associated with suicide } \\
\text { Men }: H R=2.03(0.96-4.29) \\
\text { Women: } H R=2.36(1.31-4.24)\end{array}$ \\
\hline $\begin{array}{l}\text { Cheung, } \\
2008 \text { (21) }\end{array}$ & PS & $\begin{array}{l}\text { Comm data of } \\
\text { older adults } \\
65+\end{array}$ & SARS & $\begin{array}{l}\text { SDE psychiatric profile, } \\
\text { medical profile and personal } \\
\text { issues }\end{array}$ & SD & 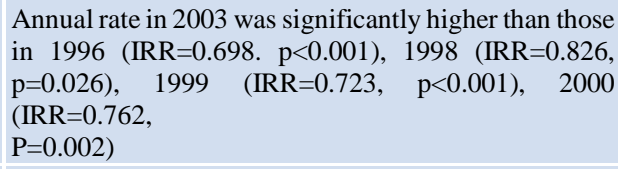 \\
\hline $\begin{array}{l}\text { Lee, } 2007 \\
(43)\end{array}$ & $\mathrm{CS}$ & $\begin{array}{l}1,226 \text { comm } \\
\text { older adults }\end{array}$ & Age & SDE & SI & $\begin{array}{l}\text { Older group }(51-75 \text { years }) \text { were more likely to consider } \\
\text { suicide }(\mathrm{OR}=2.3) \text { than the youngest age group }(<25 \\
\text { years). }\end{array}$ \\
\hline $\begin{array}{l}\text { Yip,2003 } \\
\text { (44) }\end{array}$ & $\mathrm{CS}$ & $\begin{array}{l}917 \text { older } \\
\text { adults } 60+\end{array}$ & Health Status & SDE & SD & $\begin{array}{l}\text { Number of diseases OR=1.76(1.07-2.90); vision } \\
\text { problem } \mathrm{OR}=3.34(1.24-9.04) \text {; hearing problem } \mathrm{OR}= \\
2.74(1.13-6.64) \text {; court case } \mathrm{OR}=57.42(1.29-2557.9) \\
\text { Dep OR=7.23(1.52-34.38) }\end{array}$ \\
\hline $\begin{array}{l}\text { Chi,1998 } \\
(26)\end{array}$ & CS & $\begin{array}{l}279 \text { comm } \\
\text { suicide cases } \\
\text { of older } \\
\text { adults } 60+\end{array}$ & $\begin{array}{l}\text { Gender, severe } \\
\text { or painful illness }\end{array}$ & SDE & SD & $\begin{array}{l}\text { The gender ratio: } 1.4: 1 \text {, severe or painful illness }(80 \%) \text {, } \\
\text { from terminal illness }(25 \%) \text {, had a history of receiving } \\
\text { psychiatric treatment }(25 \%) \text {.indicated suicidal idea } \\
\text { before their fatal acts }(68 \%)\end{array}$ \\
\hline $\begin{array}{l}\text { Tsoh, } \\
2005(45)\end{array}$ & $\mathrm{CC}$ & $\begin{array}{l}224 \text { older } \\
\text { adults aged } \\
65+\end{array}$ & $\begin{array}{l}\text { SDE, } \\
\text { circumstances of } \\
\text { the suicide act, } \\
\text { SA }\end{array}$ & $\begin{array}{l}\text { SDE, Circumstances of the } \\
\text { suicide act and suicide intent, } \\
\text { Psychiatric morbidities }\end{array}$ & SA & $\begin{array}{l}\text { Current major dep OR=59.2(6.4-546.6), past SA } \\
\text { OR=39.1(1.2-1,244.7), arthritis } \mathrm{OR}=22.6(3.2-157.3) \text {, } \\
\text { living with children OR=0.2(0.03-0.9), } \\
\text { conscientiousness }\end{array}$ \\
\hline $\begin{array}{l}\text { Chiu,2004 } \\
\text { (46) }\end{array}$ & $\mathrm{CC}$ & $\begin{array}{l}70 \text { suicide } \\
\text { cases and } 100 \\
\text { controls }\end{array}$ & $\begin{array}{l}\text { Mental illness, } \\
\text { history of SA, } \\
\text { health care } \\
\text { utilization }\end{array}$ & SDE & SA & $\begin{array}{l}\text { SA history }: \mathrm{OR}=39.6 \mathrm{p}<0.0001 \text {, depressive disorders } \\
\mathrm{OR}=59.24(20.68-169.65) \text {; major depression } \\
\mathrm{OR}=36.25(10.48-125.41)\end{array}$ \\
\hline \multicolumn{7}{|c|}{ Taiwan } \\
\hline $\begin{array}{l}\text { Chan,2011 } \\
\text { (47) }\end{array}$ & CS & $\begin{array}{l}3,853 \text { comm } \\
\text { older adults } \\
\text { over } 65+\end{array}$ & $\begin{array}{l}\text { Gender, income, } \\
\text { smoke, dep, } \\
\text { heart disease }\end{array}$ & $\begin{array}{l}\text { SDE, Physical Disorders, Pain } \\
\text { Symptoms }\end{array}$ & SI & $\begin{array}{l}\text { SI Predictor: Female OR=2.27(1.29-4.00), No income } \\
\text { OR=1.68(1.11- 2.53), Former smoker OR=2.05(1.09- } \\
\text { 3.85), Depressive symptoms OR=34.30(21.89- } \\
\text { 53.76), Heart disease OR=1.63(1.06-2.49) }\end{array}$ \\
\hline $\begin{array}{l}\text { Yan, } 2009 \\
\text { (15) }\end{array}$ & $\mathrm{CS}$ & $\begin{array}{l}19 \text { older } \\
\text { adults over } \\
65+\text { with SA } \\
\text { in veterans' } \\
\text { homes }\end{array}$ & $\begin{array}{l}\text { Reasons that } \\
\text { cause suicide }\end{array}$ & SDE & SI, SA & $\begin{array}{l}\text { Qualitative data indicated five major themes related to } \\
\text { suicide triggers: illness and pain, death of close } \\
\text { relatives or friends, family conflicts, disputes with } \\
\text { friends or workers, and difficulty adapting to } \\
\text { institutional life }\end{array}$ \\
\hline $\begin{array}{l}\text { Yen,2005 } \\
\text { (48) }\end{array}$ & $\mathrm{CS}$ & $\begin{array}{l}1,000 \text { older } \\
\text { adults aged } \\
65-74\end{array}$ & $\begin{array}{l}\text { Suicidal } \\
\text { thoughts }\end{array}$ & $\begin{array}{l}\text { Sex, edu, religious belief, } \\
\text { employment status, marital } \\
\text { status, living } \\
\text { arrangement, and family } \\
\text { monthly income }\end{array}$ & SI & $\begin{array}{l}\text { Dep } \mathrm{OR}=15.47 \quad(9.76-24.53) \text {, lower level of edu } \\
\mathrm{OR}=2.04(3.31-1.25) \text {, community participation in the } \\
\text { past } 6 \text { months } \mathrm{OR}=1.72(1.07-2.96)\end{array}$ \\
\hline
\end{tabular}

CS: Cross sectional study; PS: Prospective study; CC: Case control study; OR: odds ratio; RR: Relative risk; Hazard ratio; HR: IRR: Incident rate ratio. SA: Suicide attempt; SI: Suicide ideation; SD: Death by Suicide; Com: Community-dwelling; SDE: Sociodemographic variables; Edu: Education,

Dep: Depression; QOL: Quality of Life; Manuscripts are ordered according to the year of publication.

\section{Other Factors}

The list of risk factors outlined above is not exhaustive, and there is emerging evidence suggesting a range of other correlative factors. Research in Hong Kong found associations between SARS and adult suicide rate [20;21]. In addition, physical health including chronic illness and physical disabilities, were also found to increase the 
likelihood of suicide. As such, improvement in the ability to predict suicidal behavior through the continued identification of specific risk factors represents one of the most important directions for future studies in this area.

\section{Consequences Associated with Suicide}

Evidence suggests that suicide mortality increases the years of life lost and disability- adjusted life years lost (Table 4). In Taiwan, research suggests that suicide deaths in older adults account for $28.9 \%$ of total suicide deaths, representing $12.4 \%$ of the total population [22]. In Hong Kong, the total years of life lost due to suicide increased by $96.0 \%$ in 30 years, from about 9,900 years in 1981 to 19,400 years in 2001 [23].

Over time, suicide behaviors could lead to increased hospitalization, including prolonged stays in an intensive care unit, in addition to further suicide attempts, and also completed suicides (12.9-39.6 folds risk) [24]. It was reported that most suicide attempts and completed suicide deaths were associated with past histories of suicide attempt(s). In a study of elderly suicides in 279 communities, $68 \%$ indicated suicide ideation before their fatal acts [25].

\section{Interventions in Chinese Elderly Suicide}

Understanding the interactive relationship between risk and protective factors in suicidal behavior and how the interaction can be modified are challenges to suicide prevention and intervention. In addition, suicide intervention is a relatively under-researched area among Chinese elderly. There have been several suicide prevention and intervention programs initiated to serve this population, but evidence is limited on the effectiveness of prevention and intervention programs (Table 5).

The Chinese Association of Mental Health (CMHA) has recently set up a suicide prevention committee that offers counseling services over telephone hotlines. However, due to the large population size and limited man power, its effectiveness remains to be seen. The Hong Kong SAR has taken an alternate approach through the Opportunities for the Elderly Project (OEP). Reaching out to older adults with high risks of suicide through early detection and screening, OEP fosters a sense of community to care for the elderly population [26]. Another government-funded multifaceted model including treatment of depression, gatekeeper training, aftercare for suicide attempters, and care management also shows evidence of reducing suicide rates of completed suicide in old-age suicide attempters [27].

Elderly suicide intervention programs were also implemented in international Chinese communities. In
Singapore, early detection and public awareness were widely publicized through media. Primary care and specialized care for those older adults experiencing high risk factors is also provided. In addition, community support is reinforced through the Center for Enabled Living (CEL), a multi-disciplinary community case management program. The Center helps connect individuals to different community resources and also provides hotline services for the elderly in crisis [28].

\section{Discussion}

Among the Chinese elderly population factors such as, older age, female, living in rural area, depressive symptoms, previous suicide attempts, negative life events and medical complications were found to correlate strongly with suicide completion. Alternatively, high levels of self-esteem, living with children, and high Instrumental Activities of Daily Living (IADL) score were found to be protective factors. Suicide attempts could lead to hospitalization, a 12-33 fold increase in risk of completed suicide, and potential detrimental effects to loved ones.

Several issues relating to the unique risk factors in Chinese communities warrant further attention. First, with respect to age distribution in China, high incidence of older adult suicides is dissonant to important Chinese traditional values of filial obligations of respect for older adults. Confucian philosophy has strongly influenced Chinese lifestyle and social interaction for thousands of years. However, in the face of rapid industrialization, modernization and social-cultural changes brought about by economic reforms, family structures and support may undergo significant transformations, potentially resulting in older adults perceiving insufficient filial support and care. Whereas stressful life events including family conflicts were found to be an important risk factor to suicide, the importance of familial integration cannot be overemphasized. Providing a sense of community and social support to the vulnerable older adult may serve as a buffer against suicide risks.

In addition, rural older adults may be at higher risks of suicide compared to their urban counterparts. Suicide in China has been historically perceived as a final act in response to social distress [29]. Given that many believe traditional values and practices to be more deeply-rooted in the countryside, the importance of such beliefs warrants particular attention. Effects of economic reform, poverty, cultural changes and the one-child policy may all contribute to the phenomenon. 
Table 4. Consequences Associated with Suicide among Chinese Aging Population.

\begin{tabular}{|c|c|c|c|c|c|c|}
\hline $\begin{array}{l}\text { Author, } \\
\text { Year }\end{array}$ & $\begin{array}{l}\text { Study } \\
\text { Desig } \\
\text { n }\end{array}$ & Population and Setting & Predictor & Outcomes & $\begin{array}{l}\text { Confounding } \\
\text { Factors }\end{array}$ & Key Findings \\
\hline $\begin{array}{l}\text { Chi, } 2011 \\
(23)\end{array}$ & PS & Taiwan, 1997- 2007. & $\cos$ & PYLL & & $\begin{array}{l}\text { Suicide deaths in older adults account for } 28.9 \% \text { of } \\
\text { suicide deaths while representing only } 12.4 \% \text { of the } \\
\text { total population, only accounted for } 7.1 \% \text { for the } \\
\text { total PYLL. }\end{array}$ \\
\hline $\begin{array}{l}\text { Lam, } 2010 \\
(25)\end{array}$ & PS & $\begin{array}{l}265 \text { acute poisoning patients } \\
\text { admitted to an intensive care } \\
\text { unit, Hong Kong, }\end{array}$ & $\begin{array}{l}\text { Poison } \\
\text { (Intent of } \\
\text { Suicide } \\
84.9 \% \text { ) }\end{array}$ & $\begin{array}{l}\text { Hospitaliz } \\
\text { ation }\end{array}$ & $\begin{array}{l}\text { Demographic and } \\
\text { clinical } \\
\text { characteristics }\end{array}$ & $\begin{array}{l}\text { Age }>65 \text {, ICU length of stay }>1 \text { day: } O R=4.95(1.52- \\
16.13) \text {. Hospital length of stay }>3 \text { days: } O R=6.64 \\
(1.40-31.44)\end{array}$ \\
\hline $\begin{array}{l}\text { Yip, } 2005 \\
\text { (49) } \\
\end{array}$ & PS & Hong Kong 1981-2002 & $\operatorname{COS}$ & YLL & & $\begin{array}{l}\text { Older adults' suicides in all: } 25 \% \text {; disease burden } \\
\text { in YLL: less than } 6 \%\end{array}$ \\
\hline $\begin{array}{l}\text { Yip, } 2003 \\
(50)\end{array}$ & PS & Hong Kong,1981-2001 & $\cos$ & YLL & & $\begin{array}{l}\text { The total years of life lost due to suicide increased } \\
\text { by } 96.0 \% \text {, from about } 9900 \text { years (1981) to } 19,400 \\
\text { years (2001), The total share of YLL attributable to } \\
\text { suicide deaths has increased from } 3.6 \% \text { to } 8.1 \%\end{array}$ \\
\hline $\begin{array}{l}\text { Phillips, } \\
1999 \text { (51) }\end{array}$ & PS & China: 1990-1994. & $\operatorname{COS}$ & DALYs & & DALYs: over 60 y age $1.6-8,20-30$ y $2-27$ \\
\hline $\begin{array}{l}\text { Chiu,1996 } \\
\text { (52) }\end{array}$ & PS & $\begin{array}{l}55 \text { elderly age }>65 \text { with SA } \\
\text { in a psychiatric unit, Hong } \\
\text { Kong }\end{array}$ & SA & $\begin{array}{l}\text { Further } \\
\text { SA COS }\end{array}$ & & $\begin{array}{l}\text { Further SA:3.6\% } \\
\text { COS: } 5.5 \%\end{array}$ \\
\hline $\begin{array}{l}\text { Liu, } 2005 \\
(53)\end{array}$ & CS & Community data in Beijing & $\operatorname{COS}$ & DALY & & Total: 1.10, aged $>65: 1.05$ \\
\hline $\begin{array}{l}\text { Shen, } 2002 \\
\text { (54) }\end{array}$ & CS & $\begin{array}{l}333 \text { self poisoning in- } \\
\text { patients, Wu han, China }\end{array}$ & SA & $\begin{array}{l}\text { COS } \\
\text { COST }\end{array}$ & & $\begin{array}{l}\text { Aged } 60-85 \text { had the highest fatality rate of } 15.9 \% \text {, } \\
\text { and the highest average cost of }(\mathrm{P}<0.01)\end{array}$ \\
\hline $\begin{array}{l}\text { Chi, } 1998 \\
(26)\end{array}$ & CS & $\begin{array}{l}279 \text { community suicide } \\
\text { elderly age }>60 y \text {, Hong Kong }\end{array}$ & SI & COS & & $68 \%$ indicated SI before their fatal acts \\
\hline $\begin{array}{l}\text { Tsoh, } 2005 \\
\text { (45) }\end{array}$ & $\mathrm{CC}$ & $\begin{array}{l}224 \text { elderly aged }>65 y,(66 \\
\text { SA-er referred to the } \\
\text { Department of Psychiatry } \\
\text { (PWH), } 67 \text { COS-er from a } \\
\text { psychological autopsy } \\
\text { study), } 91 \text { community } \\
\text { controls, Hong Kong }\end{array}$ & SA & $\cos$ & $\begin{array}{l}\text { Socio-demographic } \\
\text { variables, } \\
\text { Circumstances of } \\
\text { the suicide act and } \\
\text { suicide intent, } \\
\text { Psychiatric } \\
\text { morbidities }\end{array}$ & Past SA: OR=32.9( $3.2-339.7)$ \\
\hline \begin{tabular}{|l|} 
Chiu, 2004 \\
(46)
\end{tabular} & $\mathrm{CC}$ & $\begin{array}{l}\text { Age }>60,70 \text { suicides }, 100 \\
\text { controls, Hong Kong }\end{array}$ & SA & $\operatorname{COS}$ & & $\mathrm{SA}: \mathrm{OR}=39.6$ \\
\hline $\begin{array}{l}\text { Phillips, } \\
2002(55)\end{array}$ & $\mathrm{CC}$ & $\begin{array}{l}519 \text { suicide adult, } 536 \\
\text { community controls, China. }\end{array}$ & $\begin{array}{l}\text { SA, other's } \\
\text { suicidal } \\
\text { behavior; }\end{array}$ & COS & $\begin{array}{l}\text { Sex, age, location } \\
\text { of residence, } \\
\text { and research site, }\end{array}$ & $\begin{array}{l}\text { Previous SA: OR=12.9(4.0-41.0); Blood relative } \\
\text { had suicidal behavior: } \mathrm{OR}=3.4(1.6-7.2) \text {, Friends or } \\
\text { associates had suicidal behavior: } \mathrm{OR}=3.3(1.9-5.8) \text {. }\end{array}$ \\
\hline
\end{tabular}

Note: CS: Cross sectional study; PS: Prospective study; CC: Case control study; COS: Complete suicide; SI: Suicide ideation; SA: Suicide attempt; OR: odds ratio; RR: Relative risk. PYLL: Potential Years of Life lost, DALYs: Disability Adjusted Life Years lost per 1,000 populations. Manuscripts are ordered according to the year of publication

Furthermore, cultural considerations in suicidal behaviors of overseas Chinese elderly immigrants should also be noted. It was reported that more than $80 \%$ of Chinese older adults were foreign born and more than $30 \%$ of Chinese older adults immigrated after the age of 60. Suffering from the loss of tradition, lack of acculturation and aging, the older Chinese population in the U.S. often lives in isolation, far away from their families in China. Hindered by cultural and language barriers, they experienced much difficulty in communication with others, let alone the ability to seek help. Therefore, it was not surprising that studies suggested that older Chinese immigrants have poorer quality of life than the national norms [30]. Other studies also pointed out that the prevalence of depressive symptoms among Chinese older adults was higher than that of general aging population in North America [31]. Coupled with the increasing social stressors and culturally valued imperatives of maintaining personal honor, older adults may consider themselves as "burden" to their families, and thus opt to suicide as means to resolve conflict. 
Table 5. Intervention on Suicide among Chinese Older Adults.

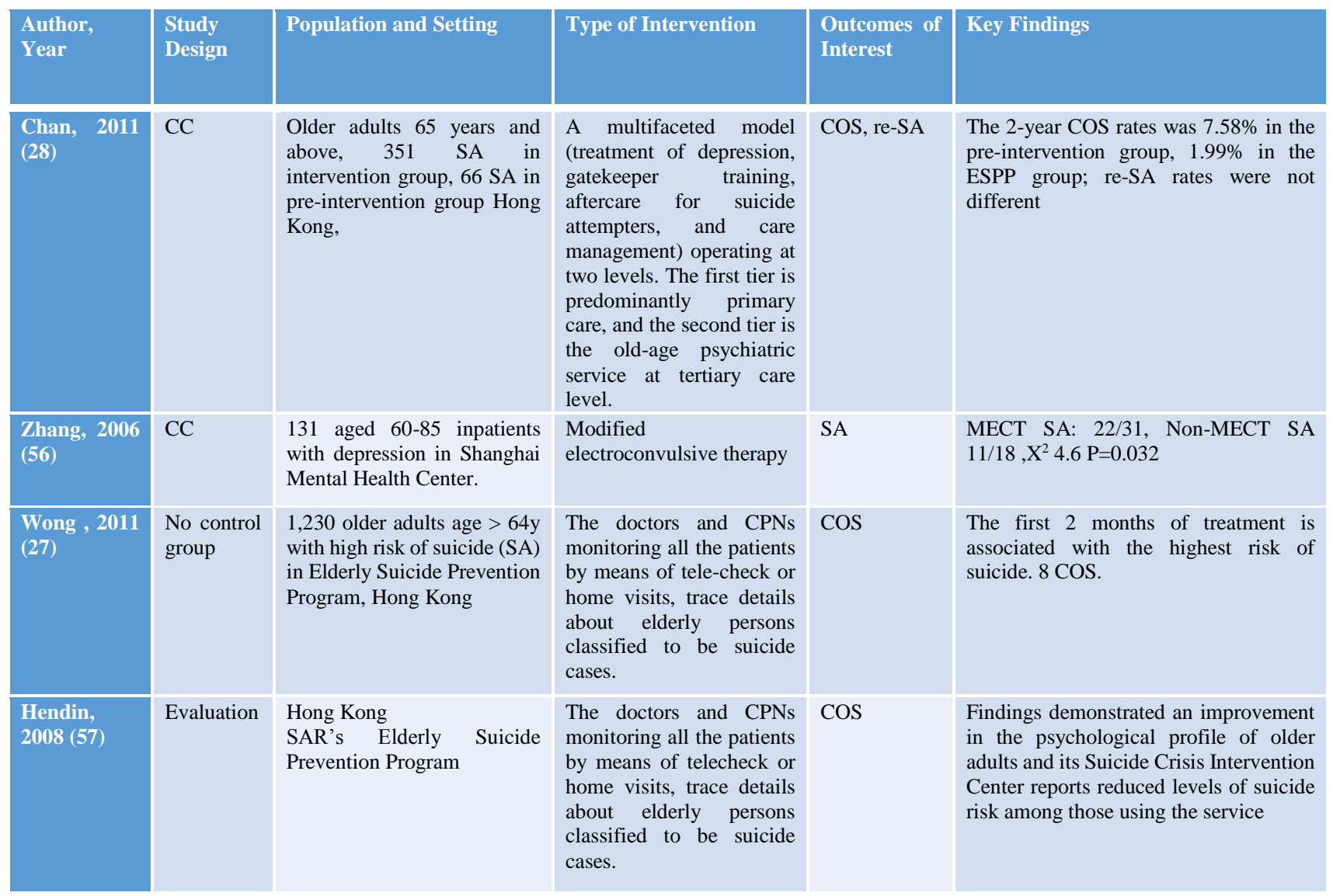

Note: CS: Cross sectional study; PS: Prospective study; CC: Case control study; COS: Complete suicide; SA: Suicide attempt; SI: Suicide ideation

\section{Implications}

Suicide among the Chinese elderly is an important public health issue. Current research highlights that elderly suicide is deeply affected by the socio, cultural, and familial contexts within which it takes place. With respect to prevention and intervention strategies, educational outreach through programs targeted to Chinese older adults with suspected suicidal inclination or risk factor of suicide, may serve as an effective measure.

Early detection and prevention of elder suicide is necessary. In addition, mental health and health professionals at the front line should consider outreach programs that are particularly designed to increase familial, social, and community support for vulnerable older adults. Medical professionals should also consider screening older patients for suicidal risk factors by integrating questions on suicidal thoughts into routine medical history questions for older adults.

These findings have important implications not only for geriatricians, but also for multiple disciplines that work with older adults with suicide risk. Other relevant disciplines such as nursing, social work, and social services agencies are also in unique positions to identify and intervene on predisposing factors that might lead to completed suicide. Direct suicide prevention strategies with culturally and linguistically sensitive services including hotlines and counseling programs should be expanded.

In addition, our review findings call for improved health and social welfare programs for the older adults, particularly for those in the rural areas and those that lack of financial support. Whereas reducing risk factors may contribute in lowering suicides, measures to improve protective factors are also critical. The prevention of elderly suicide needs to be a joint effort by family members, and communities at large. Support through ongoing family and community care relationships is necessary to improve resilience and positive aging. 


\section{Conclusion}

In sum, Chinese older adults reported the highest suicide rate in the world. This statistic is the result of multiple factors including biological, social, economic, and cultural contexts, and should be given high priority with regard to prevention, intervention and research. Rigorous research on the prevalence of suicidal ideation, attempts and plans in different Chinese aging population are urgently called for. Future longitudinal studies on the risk factors and protective factors, and adverse health consequence will be useful to devise culturally and linguistically appropriate prevention and intervention programs.

\section{Funding Support}

Dr. Dong was supported by National Institute on Aging grant (R01 AG042318, R01 MD006173, R01 AG11101 \& RC4 AG039085), Paul B.Beeson Award in Aging (K23 AG030944), The Starr Foundation, American Federation for Aging Research,John A.Hartford Foundation and Thehe Atlantic Philanthropies.

\section{References}

[1] Phillips MR, Li X, Zhang Y (2002). Suicide rates in China. Lancet, 359:835-40.

[2] Pritchard C, Baldwin DS (2002). Elderly suicide rates in Asian and English-speaking countries. Acta Psychiatr Scand, 4:271-75.

[3] Li X, Xiao Z, Xiao S (2009). Suicide among the elderly in mainland China. Psychogeriatrics, 9:6266.

[4] Liu WT \& Yu E (1985). Asian/Pacific American Elderly: Mortality differentials, health status, and use of health services. J Appl Gerontol, 4: 35-64.

[5] McCracken M, Olsen, Chen MS, Jemal A, Thun M, Cokkinides V (2007). Cancer incidence, mortality, and associated risk factors among Asian Americans of Chinese, Filipino, Vietnamese, Korean, and Japanese ethnicities. CA Cancer J Clin, 57:190205.

[6] Mui AC (1996). Depression among elderly Chinese immigrants: an exploratory study. Social Work, 41:633-45.

[7] Lee S, Tsang A, Li XY, Phillips MR, Kleinman A (2007). Attitudes toward suicide among Chinese people in Hong Kong. Suicide Life Threat Behav, 37:565-75.

[8] Lee S, Fung SC, Tsang A, Liu ZR, Huang YQ, He YL (2007). Lifetime prevalence of suicide ideation, plan,and attempt in metropolitan China. Acta Psychiatr Scand, 116:429-37.
[9] Rebholz CM, Gu D, Yang W, Chen J, Wu X, Huang J (2011). Mortality from suicide and other external cause injuries in China: A prospective cohort study. BMC Public Health, 11:56.

[10] Zhou MG, Zhang YP, Wang LJ, Huang ZJ, Michael RP, Yang GH (2004). Analysis of negative life events among 304 elderly suicide victims. Chinese Journal of Epidemiology, 25:292-95.

[11] Qin Y, Wu RZ (2002). The risk factor of suicide in female elderly depression. Health Psychology Journal, 10:51-52.

[12] Phillips MR, Yan PZ, Li JW, Hui YJ, Mai GZ (2002). Risk factors for suicide in China: a national case-control psychological autopsy study. Lancet, 360:1728-836.

[13] Phillips MR, Shen Q, Liu X, Pritzker S, Streiner D, Conner K (2007). Assessing depressive symptoms in persons who die of suicide in mainland China. $\mathbf{J}$ Affect Disord, 98:73-82.

[14] Yan CK, Yun FT, Yan CL, Yea PL (2009). Suicide experiences among institutionalized older veterans in Taiwan. The Gerontologist, 49:746-54.

[15] Gan JL, Lu CS, Yang YJ (2001). A follow-up study on risk factors of suicide attemps in senile affective disorders. Shandong Arch Psychiatry, 14:22-24.

[16] Tong Y, Phillips MR (2010). Cohort-specific risk of suicide for different mental disorders in China. Br J Psychiatry, 196:467-73.

[17] Hong CJ, Huo SJ, Yen FC, Tung CL, Pan GM, Tsai SJ (2003). Association study of a brain-derived neurotrophic-factor genetic polymorphism and mood disorders, age of onset and suicidal behavior. Neuropsychobiology, 48:186-89.

[18] Hwang JP, Yang CH, Hong CJ, Lirng JF, Yang YM, Tsai SJ (2006). Association of APOE genetic polymorphism with cognitive function and suicide history in geriatric depression. Dement and Geriatr Cogn Disord, 22:334-38.

[19] Jia CX, Zhao ZT, Hu MH, Gao LJ, Wang XT (2005). A paired case-control study on related factors to attempted suicide. Zhonghua Liu Xing Bing Xue Za Zhi, 26:339-43.

[20] Cheung YT, Chau PH, Paul SFY (2008). A revisit on older adults suicides and Severe Acute Respiratory Syndrome (SARS) epidemic in Hong Kong. Int J of Geriatri Psychiatry, 23:1231-38.

[21] Chan SM, Chiu FK, Lam CW, Leung PY, Conwell Y (2006). Elderly suicide and the 2003 SARS epidemic in Hong Kong. Int $\mathbf{J}$ of Geriatric Psychiatry, 21:113-18.

[22] Chi KL, Yip PSF, Ying YC (2011). The economic and potential years of life lost from suicide in Taiwan, 1997-2007. Crisis, 32:152-59. 
[23] Yip PSF (1997). Suicides in Hong Kong, 19811994. Soc Psychiatry Psychiatr Epidemiol, 32:24350.

[24] Lam SM, Lau AC, Yan WW (2010). Over 8 years experience on severe acute poisoning requiring intensive care in Hong Kong, China. Hum Exp Toxicol, 29:757-65.

[25] Chi I, Yip BS, Yu GK, Halliday P (1998). A study of elderly suicides in Hong Kong. Crisis, 19:35-46.

[26] Wong MM (2011). Patients committing suicide whilst under the care of the elderly suicide prevention programme of a regional hospital in Hong Kong. East Asian Arch Psychiatry, 21:17-21.

[27] Chan SS, Leung VP, Tsoh J, Li SW, Yu CS, Yu GK (2011). Outcomes of a two-tiered multifaceted elderly suicide prevention program in a Hong Kong Chinese community. Am J Geriatr Psychiatry, 19: 185-96.

[28] Ho ECL, Chiu HFK, Chong M, Yu X, Kundadak G, Kua EH (2012). Elderly suicide in Chinese populations. Asia-Pacific Psychiatry, 4:5-9.

[29] Ji J, Kleinman A, Becker AE (2009). Suicide in contemporary China: A review of China's distinctive suicide demographics in their sociocultural context. Harvard Rev Psychiatry, 9:11-12.

[30] Mui A, Kang S, Kang D, Domanski M (2007). English language proficiency and health-related quality of life among Chinese and Korean immigrant elders. Health Society, 32:119-27.

[31] Lai DWL (2004). Depression among elderly Chinese-Canadian immigrants from Mainland China. Chinese Medical Journal, 117:677-83.

[32] Xu H, Xiao S, Chen J, Liu L. (2000). Epidemiological study on committed suicide among the elderly in some urban and rural areas of Hunan province, China. Chinese Mental Health Journal, 2: 121-24.

[33] Tao RQ, Zen Z, Zhong GL, Cha WT, Liang W (2011). Case-control study of suicide ideation among the elderly in some village in Hunan. Journal of Hunan Normal University, 8:82-85.

[34] Zhang Y, Conner KR, Phillips MR (2010). Alcohol use disorders and acute alcohol use preceding suicide in China. Addict Behav, 35: 152-56

[35] Gong Y, Zhang L, Wang Z, Liang Y (2011). Pathway analysis of risk factors for severe suicidal ideation: a survey in rural China. Can J Public Health, 102:472-75.

[36] Ma X, Xiang YT, Cai ZJ, Li SR, Xiang YQ, Guo HL (2009). Lifetime prevalence of suicidal ideation, suicide plans and attempts in rural and urban regions of Beijing, China. Aust $\mathrm{N} \mathrm{Z}$ Psychiatry, 43:158-66.
[37] Sun WJ, Xu L, Chan WM, Lam TH, Schooling CM (2012). Depressive symptoms and suicide in 56,000 older Chinese: a Hong Kong cohort study. Soc Psychiatry Psychiatr Epidemiol, 47:505-14.

[38] Sun W, Schooling CM, Chan WM, Ho KS, Lam TH (2011). The association between depressive symptoms and mortality among Chinese elderly: a Hong Kong cohort study. J Gerontol A Bio Sci Med Sci, 66:459-66.

[39] Lee S, Tsang A, Li XY, Phillips MR, Kleinman A (2007). Attitudes toward suicide among Chinese people in Hong Kong. Suicide Life Threat Behav, 37: 565-75.

[40] Yip PS, Chi I, Chiu H, Chi WK, Conwell Y, Caine E (2003). A prevalence study of suicide ideation among older adults in Hong Kong SAR. Int J Geriatr Psychiatry, 18:1056-62.

[41] Tsoh J, Chiu HF, Duberstein PR, Chan SS, Chi I, Yip PS (2005). Attempted suicide in elderly Chinese persons: a multi-group, controlled study. Am J Geriatr Psychiatry, 13:562-71.

[42] Chiu HFK, Yip PSF, Chan S, Tsoh J, Kwan CW, Li SF (2004). Elderly suicide in Hong Kong- A case controlled psychological autopsy study. Acta Psychiatr Scand, 109:299-305.

[43] Chan HL, Liu CY, Chau YL, Chang CM (2011). Prevalence and association of suicide ideation among Taiwanese elderly - a population-based cross-sectional study. Chang Gung Med Journal, 34:197-204.

[44] Yen YC, Yang MJ, Yang MS, Lung FW, Shih CH, Hahn CY (2005). Suicidal ideation and associated factors among community-dwelling elders in Taiwan. Psychiatry Clin Neurosci, 59:365-71.

[45] Yip PSF, Ka YC, Law CK., Law YW (2005). Social and economic burden of suicides in Hong Kong SAR: A year of life lost perspective. Crisis, 26:15659.

[46] Yip PSF, Law CK, Law YW (2003). Suicide in Hong Kong: epidemiological profile and burden. Hong Kong Med J, 9: 419-26.

[47] Phillips MR, Hua QL, Yan PZ (1999). Suicide and social change in China. Cult Med Psychiatry, 23:25-50.

[48] Chiu HF, Lam LC, Pang AH, Leung CM, Wong CK (1996). Attempted suicide by Chinese elderly in Hong Kong. Gen Hosp Psychiatry, 18:444-47.

[49] Phillips MR (2002). Suicide rates in China 19951999. Lancet, 359:835-40.

[50] Zhang XK, Feng YF, Wu HD, Su JN (2006). Retrospective investigation on the characteristics of treatment for the elderly with depression in hospitalization. Chinese J of Clin Reh.10:4-6. 